\title{
Sentinel node biopsy in ductal carcinoma in situ (DCIS) of the breast: never justified?
}

Luca Sorrentino $^{\mathrm{a}}$ M.D., Alessandra Sartani ${ }^{\mathrm{a}}$ M.D., Daniela Bossi ${ }^{\mathrm{a}}$ M.D., Rosella Amadori ${ }^{\mathrm{b}}$ M.D., Manuela Nebuloni $^{\mathrm{c}}$ M.D. Ph.D., Marta Truffi ${ }^{\mathrm{d}}$ Ph.D., Matteo Bonzini ${ }^{\mathrm{e}}$ M.D. Ph.D., Eliana Riggio ${ }^{\mathrm{a}}$ M.D., Diego Foschi ${ }^{\mathrm{a}}$ M.D. Ph.D., Fabio Corsi ${ }^{\mathrm{a}^{*}}$ M.D. Ph.D.

\footnotetext{
a Surgery Division, Department of Clinical Sciences University of Milan, "Luigi Sacco” Hospital, via G. B. Grassi 74, 20157 Milano, Italy.

'Division of Radiology, “Luigi Sacco” Hospital, via G. B. Grassi 74, 20157 Milano, Italy.

'Department of Pathology, Department of Clinical Sciences University of Milan, "Luigi Sacco" Hospital, via G. B. Grassi 74, 20157 Milano, Italy.

¿Department of Biomedical and Clinical Sciences University of Milan, "Luigi Sacco” Hospital, via G. B. Grassi 74, 20157 Milano, Italy.
}

${ }^{\mathrm{e}}$ Centro Studi Epidemiologia e Medicina Preventiva, Department of Clinical and Experimental Medicine, Insubria University, via O. Rossi, 21100 Varese, Italy.

*Corresponding Author:

Prof. Fabio Corsi, MD

Department of Clinical Sciences, University of Milan

"Luigi Sacco" University Hospital

Via G. B. Grassi, 74

20157 - Milano, Italy

E-mail: fabio.corsi@unimi.it

Phone: $+\underline{+39.02 .3904 .3449}$

Fax: $+\underline{+39.02 .5031 .9846}$

Running Title: When to perform sentinel node biopsy in DCIS?

Keywords: DCIS; Sentinel lymph node biopsy; Upstaging; Breast cancer

\begin{abstract}
Sentinel lymph node biopsy for ductal carcinoma in situ (DCIS) of the breast is a matter of debate. Some current guidelines state that this procedure is not warranted; however, nodal involvement for DCIS patients is reported. Aim of our study was to identify preoperative features predictive of nodal involvement in DCIS patients. We have retrospectively reviewed 175 patients with a preoperative diagnosis of DCIS following a vacuum-assisted breast biopsy, and undergoing surgery with sentinel node biopsy. Variables distribution was compared between patients upstaged to invasive cancer at final pathology and patients with a confirmed DCIS, and between positive vs. negative sentinel node patients. Univariate and multivariate analyses were performed for risk of a positive node. Lymph node biopsy was positive in 13 patients $(7.4 \%)$, with 8 macrometastases $(61.5 \%)$ and 5 micrometastases (38.5\%). In these patients, Breast Imaging Reporting and Data System (BI-RADS) index $>4$ (OR 4.69, 95\%CI 1.282-17.224, $p=0.02)$, lesion extension $\geq 20 \mathrm{~mm}$ (OR 4.25, 95\%CI 1.255-14.447, $p=0.02$ ), multifocal disease (OR 4.12, 95\%CI 0.987-17.174, $p=0.05$ ), comedo type (OR 3.54, 95\%CI 1.044-11.969, $p=0.04$ ), and upstaging (OR 4.56, 95\%CI $1.080-19.249, p=0.04)$ were all predictive of nodal involvement, although upstaging could not be
\end{abstract}


predicted preoperatively. By multivariate analysis, the strongest independent factor predictive for positive sentinel node was multifocal disease (OR 5.14, 95\%CI 1.015-26.066, $p<0.05$ ). A preoperative diagnosis of DCIS, also including advanced biopsy systems such as vacuum-assisted breast biopsy, may be not always sufficient to exclude patients from sentinel node biopsy. DCIS patients with associated BI-RADS $>4$, lesion extension $\geq 20 \mathrm{~mm}$, multifocal disease or comedo type should be considered for axillary evaluation.

\section{Introduction}

Over the last decades the proportion of breast cancers diagnosed as ductal carcinoma in situ (DCIS) has progressively raised due to the extensive use of mammography screening, and currently DCIS represents about $20-25 \%$ of all breast malignancies $(1,2)$. DCIS of the breast is a pre-invasive neoplasm without periductal stromal invasion and theoretically unable to metastasize; therefore sentinel lymph node biopsy (SLNB) procedure for DCIS is controversial $(3,4)$. According to the vast majority of guidelines and position statements, SLNB is not warranted for DCIS $(4,5)$. However a substantial proportion of breast surgeons still perform SLNB in these cases, with an increasing trend over time (4-6). This discrepancy between guidelines and current clinical practice may reflect some uncertainty and a need for further research in this field, and some important clinical issues have been emerged (7). Indeed, if upstaging to invasive ductal carcinoma is found on final pathology, the patient would need a second surgical procedure to perform SLNB. Moreover, in case of mastectomy performed for extensive DCIS in which pathology on surgical specimen reveals foci of invasive disease, a second surgery for SLNB becomes mandatory but is no more possible (6, 7). DCIS is most frequently identified by the presence of a cluster of microcalcifications, a nonpalpable nodule or parenchymal distortion. Due to the nature of these lesions, the gold standard technique for histopathological diagnosis is core biopsy $(8,9)$. The main concern is that, although highly accurate, core biopsy could miss a small area of invasive carcinoma in the context of DCIS in up to $25.9 \%$ of patients, with subsequent underestimation (2). These data have a strong clinical implication, since a quarter of patients with a preoperative diagnosis of DCIS could be undertreated whenever SLNB is not performed (2). In other words, patients with a diagnosis of DCIS could be safely avoid upfront axillary evaluation from a theoretical point of view, but the problem is that a relevant proportion of DCIS are not really DCIS (2). Moreover in some DCIS subgroups, unexpectedly, distant or loco-regional recurrences have been diagnosed without finding invasive carcinoma at first surgery (10). Therefore, in some cases, DCIS could be clinically similar to small invasive cancers, and the statement that "DCIS is not lethal in itself" might need to be revisited in the next future (10). In this scenario, defining preoperative predictors of invasive disease or nodal involvement in DCIS could be helpful for a proper selection of those patients who may benefit of axillary staging. Aim of our analysis was to identify preoperative clinical, radiological or pathological variables, which could aid in proper selection of those DCIS patients that might benefit from SLNB both in case of mastectomy and breast-conserving surgery, in order to plan the most appropriate adjuvant therapy. 


\section{Materials and Methods}

\section{Case selection}

From November 2009 to July 2014 we have reviewed a continuous series of 175 patients referred to the Breast Unit of "Luigi Sacco" Hospital, who had a suspected breast lesion on mammography or ultrasound, and had undergone vacuum-assisted breast biopsy (VABB) with resulting diagnosis of pure DCIS and subsequent surgery. Patients with microinvasive disease demonstrated on VABB histology were excluded from the study. In all cases VABB was performed by the same radiologist with a 11-Gauge needle. Biopsy has been performed in 167 patients $(95.4 \%)$ under stereotactic guidance using a Fischer Mammotome Plus S table (Biopsy System 85,201 6-1, Fischer Imaging Corporative, Denver, CO), while in 8 patients (4.6\%) under sonographic guidance using a Hitachi H21 ultrasound (Hitachi, Tokyo, Japan; 7.5-MHz probe). Twelve specimens from each patient were collected, fixed in $10 \%$ buffered formalin and sent for histopathological examination by standard hematoxylin-eosin with p63 staining in order to assess microinvasive disease. Only patients with a preoperative histopathology of pure DCIS without microinvasive disease and without ultrasound evidence of lymphadenopathy ( $\mathrm{pT}$ Tis $\mathrm{cN} 0$ ) were included in the analysis.

Surgery was performed on all the patients by the same surgical team. In 145 cases $(82.9 \%)$ a lumpectomy with concurrent SLNB was performed, while in 30 cases $(17.1 \%)$ mastectomy with SLNB was necessary due to multifocal or extensive $(>5 \mathrm{~cm})$ disease. Mastectomies and lumpectomies were both included in the analysis to investigate predictors of upstaging or nodal involvement beyond the type of surgery. During the period analyzed by the study, all patients with a preoperative diagnosis of DCIS underwent SLNB. All SLNs were entirely processed intraoperatively by One-Step Nucleic acid Amplification (OSNA) technique (Sysmex, Kobe, Japan); SLN has never been assessed by histopathology with hematoxilin-eosin staining or immunohistochemistry (11). Briefly, the SLN was homogenized with $4 \mathrm{~mL}$ of a lysis buffer solution (Lynorhag, Sysmex, Kobe, Japan) for 90 seconds, and centrifuged at $10000 \mathrm{~g}$ at room temperature for 60 seconds. A 2 uL sample of the supernatant was analyzed with the RD-100i system (Sysmex, Kobe, Japan), an automated mRNA amplification detection system using a reverse transcription loop-mediated isothermal amplification method, and with a reagent kit (LynoampBC, Sysmex, Kobe, Japan). Upon precipitation of magnesium pyrophosphate, a product of the reaction, the resulting change in turbidity was in turn correlated to the CK19 mRNA copy number of the original lysate via a standard curve (established previously) with three calibrators containing different CK19 mRNA concentrations. A standard positive control sample containing 5000 copies of CK19 mRNA and a negative control sample containing no copies of CK19 mRNA were used for validation in each assay. The number of CK19 mRNA copies/uL was calculated, and each analyzed SLN was assigned to one of the three categories: negative if containing $<250 \mathrm{mRNA}$ copies/uL, micrometastasis $(+)$ if the amount was $250-5000$ mRNA copies/uL, and macrometastasis $(++)$ if ranging $>5000 \mathrm{mRNA}$ copies/uL In all cases in which a macrometastasis has been intraoperatively diagnosed, axillary dissection was performed, while for micrometastases no further axillary surgery has been executed in our Institute from 2013, according to the updated St. Gallen guidelines regarding the management of the axilla (12). In case of positive SLN, an extensive histopathological analysis with p63 staining including all the surgical specimen was carried out to verify the presence of misdiagnosed foci of invasive disease. 


\section{Study design}

Baseline clinical, radiological and pathological characteristics were retrospectively collected for all patients. A discordance analysis between VABB histology and surgical specimen was performed to verify which patients were upstaged to invasive carcinoma on final pathology. Patients were subsequently divided in two groups: those in which agreement between VABB results and final pathology was observed and those in which, on the contrary, upstaging to invasive disease occurred. A comparison between the two groups was performed to assess variables distribution and to identify possible preoperative features related to upstaging to invasive disease. Patients were further divided into two additional groups according to the SLNB results: those with a positive SLNB for micro- or macrometastasis and those with a negative SLNB, including isolated tumor cells. A subsequent comparison between these two groups was performed to identify preoperative predictors of positive SLNB. Finally, univariate and multivariate analyses were carried out for both risk of upstaging to invasive ductal carcinoma and risk of nodal involvement.

\section{Statistical analysis}

Baseline features of patients were reported as means \pm standard deviations in case of continuous variable or as absolute numbers and percentages in case of categorical data. Categorical variables were compared using the chi-square or Fisher exact test, whereas continuous variables were compared using the Student $t$ test. Univariate and multivariate analyses were performed by logistic regression analysis. A $p$ value $<0.05$ was considered significant. Statistical analysis was carried out using STATA software, version 11.0 (StataCorp, Austin, TX). 


\section{Results}

Predictive factors for upstaging to invasive disease

The overall agreement between VABB and final pathology on surgical specimen resulted to be $92.6 \%(162 / 175$ cases $)$, while 13 patients $(7.4 \%)$ were upstaged to invasive disease. No significant differences in variables distribution were found between these two groups of patients, as reported in Table 1. However, finding of a mass on mammography was more frequent for upstaged tumors $(15.4 \%$ vs. $1.9 \%, p=0.064)$, while microcalcifications were more common in DCIS $(97.5 \%$ vs. $84.6 \%, p=0.064$ ). The proportion of estrogen receptor (ER)-negative and progesterone receptor (PR)-negative lesions was higher in upstaged patients: ER expression was negative in $38.5 \%$ vs. $16 \%$ cases $(p=0.06$ ), while PR expression was unexpressed in $53.8 \%$ vs. $26.5 \%$ patients $(p=0.057$ ).

\section{Sentinel node biopsy results and axillary status}

In 162 cases $(92.6 \%)$ SLNB was negative, while 13 patients $(7.4 \%)$ had metastatic disease distributed as follows: 8 macrometastases $(61.5 \%)$ and 5 micrometastases $(38.5 \%)$. Axillary dissection was performed on 11 patients $(84.6 \%)$, while in 2 recent cases $(15.4 \%)$ in which the SLNB revealed micrometastasis, axillary dissection has been avoided. Among axillary dissections, 4 patients $(36.4 \%)$ had metastasis only in the sentinel node, while in 7 cases $(63.6 \%)$ other lymph nodes were involved by metastatic disease on final pathology. In particular, 6 patients $(54.5 \%)$ had $\geq 3$ metastatic nodes, and 1 patient $(9.1 \%)$ had 2 metastatic nodes. All these data are reported in Table 2 .

\section{Predictive factors for positive sentinel node biopsy}

Breast Imaging Reporting and Data System (BI-RADS) score was $\geq 4$ in a higher proportion of patients with positive SLNB (30.8\% vs. 8.6\%, $p=0.015)$, while BI-RADS 3 was present more frequently in negative SLNB patients $(21 \%$ vs. $0 \%, p=0.015)$. Lesion extension measured by mammography was also different in the two groups, being $\geq 20 \mathrm{~mm}$ in $69.2 \%$ of positive SLNB patients vs. $34.6 \%$ for the other group $(p=0.017)$. Multifocal disease was observed on mammography in $23.1 \%$ of positive SLNB and in only $6.8 \%$ of negative SLNB, $p=0.037$ ). Finally, comedo type of DCIS was found on VABB more commonly than other DCIS subtypes in a significant percentage of positive SLNB patients $(69.2 \%$ vs. $38.9 \%, p=0.041)$. The proportion of upstaging was significantly higher in positive SLNB group of patients: $23.1 \%$ vs. $6.2 \%, p=0.025$. The distribution of all variables is reported in Table 3.

\section{Predictive factors for positive sentinel node resulting from univariate or multivariate analysis}

The univariate analysis for risk of nodal involvement confirmed all predictive factors identified. In particular, BI-RADS index $>4$ was strongly associated with positive SLNB (OR 4.69, 95\%CI $1.282-17.224, p=0.02)$. Other significant predictors for positive SLNB were: lesion extension $\geq 20$ $\mathrm{mm}$ (OR 4.25, 95\% CI 1.255-14.447, $p=0.02$ ), multifocal disease on mammography (OR 4.12, 95\%CI 0.987-17.174, $p=0.05$ ), and comedo type DCIS (OR 3.54, 95\%CI 1.044-11.969, $p=0.04$ ), as reported in Table 4. Upstaging to invasive disease at final pathology was recognized as another significant predictor of nodal involvement (OR 4.56, 95\% CI 1.080-19.249, $p=0.04$ ).

The multivariate analysis for risk of nodal involvement was performed including the predictors 
identified upon univariate analysis, except upstaging to invasive disease, since this variable cannot be assessed preoperatively (see Table 5). BI-RADS $>4$, lesion extension $\geq 20 \mathrm{~mm}$ and comedo DCIS were all confirmed to be associated with positive SLNB, with adjusted ORs equal to 2.76, 2.23 and 3 , respectively. Multifocal disease on mammography (OR 5.14, 95\%CI 1.015-26.066, $p<0.05$ ) emerged as the only independent predictive factor of nodal involvement.

\section{Discussion}

SLNB in DCIS is a debated procedure $(4,5)$. Indeed, various studies demonstrated a widely variable proportion of DCIS patients with SLNB positive for metastatic disease, ranging from $1 \%$ to $22 \%(13-17)$. Therefore, the role of SLNB procedure in such patients remains controversial. In a large meta-analysis of 22 studies, a rate of 7.4\% SLN metastases in DCIS patients was reported and considered rather low (13). Therefore, both the updated National Comprehensive Cancer Network (NCCN) and the American Society of Clinical Oncology (ASCO) guidelines recommend not to perform SLNB in DCIS patients undergoing breast-conserving surgery $(4,5)$. However, SLNB is mandatory in DCIS patients in which mastectomy is required (17.1\% of our series), because in case of upstaging to invasive disease a second surgery for SLNB would be complicated $(4,5)$.

Despite such guidelines, many breast surgeons still prefer to evaluate the axillary status in DCIS patients. In Canada $61.1 \%$ of DCIS patients undergoing lumpectomy and $95 \%$ of those undergoing mastectomy receive a SLNB, while in the United States these rates are $17.7 \%$ and $63 \%$ respectively, with an increased use of SLNB for mastectomy patients from 2006 to $2012(7,18)$. A recent study from the United Kingdom showed that axillary surgery was performed in $26.3 \%$ of DCIS patients in which the diagnosis was confirmed on final pathology, raising to $81.9 \%$ in case of 
mastectomy (19). Moreover, an international survey on DCIS management demonstrated that 35\% of DCIS patients undergoing breast-conserving surgery also received SLNB (6). Interestingly, a recent retrospective analysis on the Surveillance, Epidemiology, and End Results (SEER) registry performed on DCIS patients managed between 1991 and 2010, showed a significant increase in performing SLNB, from $9.7 \%$ to $67.1 \%$ for mastectomies and from $1.4 \%$ to $17.8 \%$ for lumpectomies (20). The sum of these observations is clinically relevant, and reflects a certain uncertainty for breast surgeons on the standard management of the axilla in DCIS patients. Hence, rational selection criteria for DCIS patients undergoing breast surgery which could benefit of SLNB may be required. Notably, in our analysis both mastectomies and breast-conserving surgery have been included, therefore our findings could be applied to both situations. Indeed an increasing evidence and various guidelines support upfront SLNB in case of mastectomy, but a wide variability exists among breast surgeons about this point: therefore rational criteria also for these patients could be helpful.

In our series, we report nodal involvement in $7.4 \%$ of cases, according to previously published literature. About two third of these patients had macrometastatic disease; notably, in $63.6 \%$ of axillary dissections additional lymph nodes were found to be involved on final pathology, and in $54.5 \%$ of cases $(6 / 11) \geq 3$ nodes have reported to be metastatic. Considering only macrometastatic disease as clinically significant, we concluded that $4.6 \%$ of patients ( 8 on 175) could have been understaged, and potentially undertreated, if surgeons had followed the majority of current clinical practice avoiding SLNB. Knowledge about the nodal status in these patients has allowed us to properly address adjuvant chemotherapy, which otherwise would not have been offered due a preoperative diagnosis of DCIS. Axillary surgery has been profoundly revisited after publication of the ACOSOG Z0011 and the IBCSG 23-01 clinical trials $(12,21,22)$. However, the presence of multiple macrometastases in axillary nodes still requires axillary dissection and points out the need for adjuvant chemotherapy and tighter follow up. Moreover, when $>3$ lymph nodes are involved, adjuvant regional nodes radiation therapy could be mandatory (23). Most of published series on this topic have reported a very low rate of macrometastases, thus strengthening the idea that SLNB is not necessary for DCIS patients $(13,24-26)$. Conversely, in our series the majority of patients had a macrometastasis, suggesting that caution should be used before definitively abandoning SLNB for all DCIS patients. Certainly the largest part of patients with a preoperative diagnosis of DCIS could be safely spared of SLNB without a significant impact on their subsequent treatment, but in highrisk cases SLNB could be still necessary.

Intriguingly, in 10 patients with a positive sentinel node a pure DCIS has been confirmed on final histology, although theoretically DCIS cannot metastatize (Table 2). This fact has been largely reported in literature on this topic, and three different explanations could be hypothesized for this phenomenon. First, foci of invasive ductal carcinoma could have been missed by the pathologist on tissue sampling of extensive surgical specimens, such as in case of microinvasive disease on a mastectomy (2). Second, it was recently reported that epithelial cells could migrate by lymphatic flux from tumor to the sentinel node, especially after invasive procedures such as breast biopsy; this fact has been associated to the diagnosis of axillary involvement in pure DCIS (15). Third, it was recently pointed out that a particular subset of DCIS lesions could have unknown metastatic potentital due to a peculiar biological behavior; this statement was assumed after the observation that in some cases a distant recurrence was encountered in patients previously treated for DCIS, without any evidence of invasive cancer (10). A higher rate of macrometastases has been encountered compared to published literature, but this could be related to the use of a molecular technique with high accuracy for sentinel node evaluation, while other authors have examined the sentinel node by frozen section or imprint cytology or histopathological assessment, which involves a wide range of variability (27). Certainly the use of a molecular technique to assess SLN for DCIS lesions is debatable, due to the higher rate of occult metastases without clinical significance, such as isolated tumor cells or micrometastases. However, in our series isolated tumor cells were not considered, and most of positive SLNs were macrometastatic, frequently with more than 2 involved nodes (see Table 2). Therefore SLN assessment by molecular techniques could avoid the great variability showed by other methods reducing false negative cases, while false positive with small tumor deposits should be correctly interpreted and managed by surgeons for a proper decision making on the axilla.

Our findings do not support upfront SLNB for DCIS, since in $95.4 \%$ of cases this procedure has not provided useful information for subsequent adjuvant management. However, selection criteria are 
advocated to sort the remaining $4.6 \%$ of DCIS patients, which show high likelihood of macrometastatic involvement of more than 2 axillary nodes and could benefit of axillary evaluation.

In our series, BI-RADS was associated to SLN metastases, since a score $>4$ was found more frequently in positive SLN patients (OR 4.69, $p=0.02$ ). BI-RADS score is a well-known predictor for invasiveness and, theoretically, this could explain why a higher BI-RADS may be related with SLN metastasis $(2,8)$. Lesion extension $\geq 20 \mathrm{~mm}$ and multifocal disease were two other identified factors related to positive SLNB (OR 4.25, $p=0.02$ and OR 4.12, $p=0.05$, respectively). This is probably due to an increased risk of misdiagnosed microinvasive disease missed by $\operatorname{VABB}(14,27)$. Indeed, 2 of 3 patients with a positive SLNB and with upstaged disease showed lesion size $\geq 20 \mathrm{~mm}$ and multifocal disease, both conditions in which VABB sampling could easily miss invasive cancer (see Table 2). However, on multivariate analysis BI-RADS $>4$ and lesion extension $\geq 20 \mathrm{~mm}$ resulted to be confounding factors, since multifocal disease was the only significant independent predictor of positive SLNB identified, with an adjusted OR of 5.14. This highlights the relevance of possible occult invasive disease on SLNB status. Other authors have previously demonstrated that diffuse DCIS and residual lesion on mammography after VABB are all significantly correlated with upstaging and nodal involvement $(9,27)$. Finally, we have identified the presence of comedo type DCIS, a possible precursor of basal-like breast cancer, as a predictive factor for nodal involvement (OR 3.54, $p=0.04)$, while other studies have correlated comedonecrosis only to upstaging (28-31).

As expected, upstaging to invasive disease on final pathology was significantly more frequent in positive SLNB patients $(23.1 \%$ vs. $6.2 \%, p=0.025)$. Univariate analysis revealed that upstaging was the strongest predictive factor for axillary metastases after BI-RADS $>4$, with an OR equal to 4.56 $(p=0.04)$. The presence of SLN metastases in DCIS patients has been mainly associated to upstaging, but in our series only $23.1 \%$ of patients with a positive SLNB was upstaged to invasive carcinoma. This suggests that other predictors need to be investigated beyond upstaging. Moreover, in our series no variable could predict missed invasive cancer (see Table 1). This was probably due to the very low rate of upstaging $(7.4 \%)$ with consequent limitations in comparison between groups. In similar retrospective studies, recognized predictors of invasiveness were the presence of a mammographic or palpable mass, extensive microcalcifications $>20 \mathrm{~mm}$, and solid type of DCIS $(14,28)$.

Remarkably, the use of VABB during the diagnostic phase showed a high agreement with final pathology, with a low percentage of upstaging compared to literature, in which up to $26-33.6 \%$ of DCIS diagnosed on core biopsy upstaged to invasive disease on final excision $(2,14,28)$. Some authors have underlined the importance of the number of VABB samples, since with 3 to 5 samples only a higher underestimation rate was observed $(14,28,32)$. These data could explain the low upstaging rate encountered in our series since we perform a 11-Gauge VABB with 12 samples as a standard procedure. Based on such considerations, it is even more surprising that we encountered an axillary metastases rate as high as $7.4 \%$. This observation supports two considerations: 1 ) some DCIS lesions could have peculiar behaviors similar to small invasive lesions; 2) missing foci of invasive ductal carcinoma could be more frequent than expected, also with advanced biopsy systems such as VABB and despite an accurate histopathological analysis processing the whole surgical specimens with p63 staining. Therefore, a DCIS diagnosed on VABB is not itself a sufficient condition to safely exclude the need for SLNB (10). 


\section{Conclusions}

The role of SLNB in DCIS patients remains controversial, but routine abstention may increase the risk of understaging and subsequent undertreatment of some patients. Current clinical management of the axilla is based on the assumption that all DCIS diagnosed preoperatively are pure DCIS without the possibility to metastatize. The presence of macrometastatic axillary nodes in DCIS would change the subsequent adjuvant strategy, and even micrometastases could be relevant in order to plan a proper follow up. Our data suggest that BI-RADS $>4$, lesion size $\geq 20 \mathrm{~mm}$, multifocal disease and comedo type could identify a particular subgroup of DCIS lesions with higher risk for positive SLNB, in which axillary evaluation is needed to define the most appropriate clinical management and follow-up.

\section{Acknowledgements and Conflict of Interest}

This study was not funded. All the authors reported no competing interests, no conflict of interest to be disclosed, and no acknowledgements.

\section{References}

1. Siziopikou KP. Ductal carcinoma in situ of the breast: current concepts and future directions. Arch Pathol Lab Med 2013;137:462-466.

2. Brennan ME, Turner RM, Ciatto S, et al. Ductal carcinoma in situ at core-needle biopsy: meta-analysis of underestimation and predictors of invasive breast cancer. Radiology 2011;260:119-28.

3. Jaffer S, Bleiweiss IJ. Histologic classification of ductal carcinoma in situ. Microsci Res Tech 2002;59:92-101.

4. Lyman GH, Temin S, Edge SB, et al. Sentinel lymph node biopsy for patients with earlystage breast cancer: American Society of Clinical Oncology clinical practice guideline update. J Clin Oncol 2014;32:1365-1383.

5. National Comprehensive Cancer Network Clinical Practice Guidelines in Oncology. Breast Cancer: Version $\quad 2015 . \quad$ Available: http://www.nccn.org/professionals/physician_gls/PDF/breast.pdf. (accessed March 1, 2015). 
6. Ponti A, Lynge E, James T, et al. International variation in management of screen-detected ductal carcinoma in situ of the breast. Eur J Cancer 2014;50:2695-2704.

7. Coromilas EJ, Wright JD, Huang Y, et al. The influence of hospital and surgeon factors on the prevalence of axillary lymph node evaluation in ductal carcinoma in situ. JAMA Oncol 2015;1:323-32.

8. Schulz S, Sinn P, Golatta M, et al. Prediction of underestimated invasiveness in patients with ductal carcinoma in situ of the breast on percutaneous biopsy as rationale for recommending concurrent sentinel lymph node biopsy. Breast 2013;22:537-42.

9. Trentin C, Dominelli V, Maisonneuve P, et al. Predictors of invasive breast cancer and lymph node involvement in ductal carcinoma in situ initially diagnosed by vacuumassisted breast biopsy: experience of 733 cases. Breast 2012;21:635-40.

10. Narod SA, Iqbal J, Giannakeas V, et al. Breast cancer mortality after a diagnosis of ductal carcinoma in situ. JAMA Oncol 2015;1:888-96.

11. Sagara Y, Ohi Y, Matsukata A, et al. Clinical application of the one-step nucleic acid amplification method to detect sentinel lymph node metastasis in breast cancer. Breast Cancer 2013;20:181-6.

12. Goldhirsch A, Winer EP, Coates AS, et al. Personalizing the treatment of women with early breast cancer: highlights of the St Gallen International Expert Consensus on the Primary Therapy of Early Breast Cancer 2013. Ann Oncol 2013;24:2206-23.

13. Ansari B, Ogston SA, Purdie CA, et al. Meta-analysis of sentinel node biopsy in ductal carcinoma in situ of the breast. Br J Surg 2008;95:547-54.

14. Han JS, Molberg KH, Sarode V. Predictors of invasion and axillary lymph node metastasis in patients with a core biopsy diagnosis of ductal carcinoma in situ: An analysis of 255 cases. Breast J 2011;17:223-9.

15. Francis AM, Haugen CE, Grimes LM, et al. Is sentinel lymph node dissection warranted for patients with a diagnosis of ductal carcinoma in situ? Ann Surg Oncol 2015;22:4270-9.

16. Prendeville S, Ryan C, Feeley L, et al. Sentinel lymph node biopsy is not warranted following a core needle biopsy diagnosis of ductal carcinoma in situ (DCIS) of the breast. Breast 2015;24:197-200.

17. Zetterlund L, Stemme S, Arnrup H, et al. Incidence of and risk factors for sentinel lymph node metastasis in patients with a postoperative diagnosis of ductal carcinoma in situ. $\mathrm{Br} J$ Surg 2014;101:488-94.

18. Chin-Lenn L, Mack LA, Temple W, et al. Predictors of treatment with mastectomy, use of sentinel lymph node biopsy and upstaging to invasive cancer in patients diagnosed with breast ductal carcinoma in situ (DCIS) on core biopsy. Ann Surg Oncol 2014;21:66-73.

19. Nicholson S, Hanby A, Clements K, et al. Variations in the management of the axilla in screen-detected Ductal Carcinoma In Situ: Evidence from the UK NHS Breast Screening Programme audit of screen detected DCIS. Eur J Surg Oncol 2015;41:86-93.

20. Worni M, Akushevich I, Greenup R, et al. Trends in treatment patterns and outcomes for ductal carcinoma in situ. J Natl Cancer Inst 2015;107 (Epub ahead of print).

21. Giuliano AE, McCall L, Beitsch P, et al. Locoregional recurrence after sentinel lymph node dissection with or without axillary dissection in patients with sentinel node metastases: the American College of Surgeons Oncology Group Z0011 randomized trial. Ann Surg 2010;252:426-32.

22. Galimberti V, Cole BF, Zurrida S, et al. Axillary dissection versus no axillary dissection in patients with sentinel-node micrometastases (IBCSG 23-01): a phase 3 randomised controlled trial. Lancet Oncol 2013;14:297-305. 
23. Harris JR, Halpin-Murphy P, McNeese M, et al. Consensus statement on postmastectomy radiation therapy. Int J Radiat Oncol Biol Phys 1999;44:989-990.

24. Morrow M, Katz SJ. Addressing overtreatment in DCIS: What should physicians do now? $J$ Natl Cancer Inst 2015;107 (Epub ahead of print)

25. Mitchell KB, Kuerer H. Ductal carcinoma in situ: treatment update and current trends. Curr Oncol Rep 2015;17:48.

26. De Boer M, van Deurzen CH, van Dijck JA, et al. Micrometastases or isolated tumor cells and the outcome of breast cancer. N Engl J Med 2009;361:653-63.

27. Sakr R, Bezu C, Raoust I, et al. The sentinel lymph node procedure for patients with preoperative diagnosis of ductal carcinoma in situ: risk factors for unsuspected invasive disease and for metastatic sentinel lymph nodes. Int J Clin Pract 2008;62:1730-5.

28. Osako T, Iwase T, Ushijima M, et al. Incidence and prediction of invasive disease and nodal metastasis in preoperatively diagnosed ductal carcinoma in situ. Cancer Sci 2014;105:57682.

29. Yi M, Krishnamurthy S, Kuerer HM, et al. Role of primary tumor characteristics in predicting positive sentinel lymph node in patients with ductal carcinoma in situ or microinvasive breast cancer. Am J Surg 2008;196:81-7.

30. Yen TW, Hunt KK, Ross MI, et al. Predictors of invasive breast cancer in patients with an initial diagnosis of ductal carcinoma in situ: a guide to selective use of sentinel lymph node biopsy in management of ductal carcinoma in situ. J Am Coll Surg 2005;200:516-26.

31. Shekhar MP, Kato I, Nangia-Makker P, et al. Comedo-DCIS is a precursor lesion for basallike breast carcinoma: identification of a novel p63/Her2/neu expressing subgroup. Oncotarget 2012; 4:231-41.

32. Rutstein LA, Johnson RR, Poller WR, et al. Predictors of residual invasive disease after core needle biopsy diagnosis of ductal carcinoma in situ. Breast $J$ 2007;13:251-7.

\section{Tables}

Table 1

Distribution of baseline features in confirmed DCIS and upstaged patients at final pathology

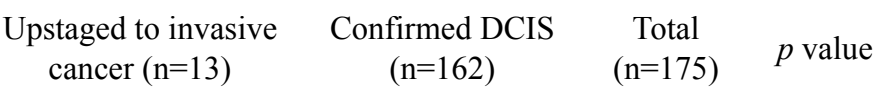

$\begin{array}{lllll}\text { Age at diagnosis (years) } & 59.7( \pm 8.5) & 59.8( \pm 9.8) & 59.8( \pm 9.8) & 0.97\end{array}$

$\begin{array}{lcccc}\text { BI-RADS } & & & & \\ 3 & 1(7.7 \%) & 33(20.4 \%) & 34(19.4 \%) & 0.723 \\ 4 & 11(84.6 \%) & 112(69.1 \%) & 123(70.3 \%) & \\ 5 & 1(7.7 \%) & 17(10.5 \%) & 18(10.3 \%) & \end{array}$

\begin{tabular}{|c|c|c|c|c|}
\hline $\begin{array}{l}\text { Mammographic } \\
\text { findings }\end{array}$ & & & & \\
\hline Microcalcifications & $11(84.6 \%)$ & $158(97.5 \%)$ & $169(96.6 \%)$ & 0.064 \\
\hline Parenchymal distorsion & $0(0.0 \%)$ & $1(0.6 \%)$ & $1(0.6 \%)$ & \\
\hline Nodule & $2(15.4 \%)$ & $3(1.9 \%)$ & $5(2.8 \%)$ & \\
\hline
\end{tabular}


Mean (mm)

$<20 \mathrm{~mm}$

$\geq 20 \mathrm{~mm}$

\section{Complete lesion}

removal at $\mathrm{VABB}$

Yes

No

\section{Multifocal disease}

Yes

$2(15.4 \%)$

No

$11(84.6 \%)$

\section{Estrogen receptors}

Negative

Positive

Unknown

\section{Progesterone receptors}

Negative

Positive

Unknown

\section{Ki67 index}

$\leq 14 \%$

$>14 \%$

Unknown

\section{Grade on VABB}

DIN I

DIN II

DIN III

\section{Central necrosis}

Yes

$5(38.5 \%)$

$8(61.5 \%)$

No

\section{Type of DCIS}

Comedo

Cribriform

Papillary

Solid

Others/Mixed

Time between VABB and surgery (days)

$6(46.2 \%)$

$5(38.5 \%)$

$8(61.5 \%)$

$0(0.0 \%)$

$7(53.8 \%)$

$6(46.2 \%)$

$0(0.0 \%)$

$6(46.2 \%)$

$7(53.8 \%)$

$0(0.0 \%)$

$3(23.1 \%)$

$7(53.8 \%)$

$3(23.1 \%)$

$5(38.5 \%)$

$0(0.0 \%)$

$2(15.4 \%)$

$0(0.0 \%)$

$6(46.1 \%)$
$24.1( \pm 18.8)$

$56(34.6 \%)$

$61(34.9 \%)$

0.769

$106(65.4 \%) \quad 114(65.1 \%)$

$$
12(7.4 \%)
$$$$
14(8 \%)
$$$$
0.278
$$$$
150(92.6 \%) \quad 161(92 \%)
$$

$\begin{array}{cc}43(26.5 \%) & 50(28.6 \%) \\ 115(71 \%) & 121(69.1 \%) \\ 4(2.5 \%) & 4(2.3 \%)\end{array}$

$74(45.7 \%)$

$80(45.7 \%) \quad 1$

$84(51.8 \%)$

$91(52 \%)$

$4(2.5 \%)$

$4(2.3 \%)$

$\begin{array}{lll}20(12.3 \%) & 23(13.1 \%) & 0.42 \\ 109(67.3 \%) & 116(66.3 \%) & \\ 33(20.4 \%) & 36(20.6 \%) & \end{array}$

$78(48.1 \%)$

$83(47.4 \%)$

0.573

$84(51.9 \%) \quad 92(52.6 \%)$

$67(41.3 \%)$

$72(41.2 \%)$

1

$15(9.3 \%)$

$15(8.6 \%)$

$15(9.3 \%)$

$17(9.7 \%)$

$2(1.2 \%)$

$2(1.1 \%)$

$63(38.9 \%)$

$69(39.4 \%)$

$45.1( \pm 21.4)$

$52.6( \pm 26.6)$

$44,5( \pm 20.9)$

0.189

$D C I S$ ductal carcinoma in situ, BI-RADS Breast Imaging Reporting and Data System, $V A B B$ vacuum-assisted breast biopsy, $D I N$ ductal intraepithelial neoplasia. 
Table 2

Baseline features and axillary status of patients with a positive SLNB

\begin{tabular}{|c|c|c|c|c|c|c|c|c|c|}
\hline Age & $\begin{array}{c}\text { Lesion } \\
\text { extension }\end{array}$ & $\begin{array}{l}\text { BI- } \\
\text { RADS } \\
\text { score }\end{array}$ & $\begin{array}{c}\text { Multifocal } \\
\text { disease }\end{array}$ & $\begin{array}{l}\text { Type of } \\
\text { DCIS }\end{array}$ & $\begin{array}{l}\text { Upstaging } \\
\text { to invasive } \\
\text { disease }\end{array}$ & $\begin{array}{l}\text { Type of } \\
\text { surgery }\end{array}$ & SLNB & $\begin{array}{c}\text { Axillary } \\
\text { dissection }\end{array}$ & $\begin{array}{c}\text { Positive } \\
\text { nodes }\end{array}$ \\
\hline 42 & 5 & 4 & No & Comedo & No & BCS & Macromet & Yes & $3 / 12$ \\
\hline 61 & 70 & 5 & No & Comedo & No & $\mathrm{TM}$ & Micromet & Yes & $1 / 14$ \\
\hline 52 & 20 & 4 & Yes & Comedo & Yes & BCS & Macromet & Yes & $4 / 13$ \\
\hline 64 & 12 & 4 & Yes & Mixed & No & $\mathrm{TM}$ & Micromet & Yes & $1 / 16$ \\
\hline 62 & 30 & 4 & Yes & Mixed & Yes & $\mathrm{TM}$ & Macromet & Yes & $4 / 12$ \\
\hline 50 & 40 & 4 & No & Mixed & No & $\mathrm{BCS}$ & Macromet & Yes & $2 / 12$ \\
\hline 69 & 20 & 5 & No & Comedo & No & $\mathrm{BCS}$ & Micromet & Yes & $1 / 14$ \\
\hline 68 & 60 & 5 & No & Comedo & No & $\mathrm{TM}$ & Macromet & Yes & $3 / 13$ \\
\hline 60 & 15 & 4 & No & Papillary & Yes & BCS & Macromet & Yes & $1 / 20$ \\
\hline 74 & 40 & 4 & No & Comedo & No & $\mathrm{BCS}$ & Macromet & Yes & $3 / 13$ \\
\hline 52 & 20 & 5 & No & Comedo & No & $\mathrm{BCS}$ & Micromet & No & - \\
\hline 74 & 7 & 4 & No & Comedo & No & $\mathrm{BCS}$ & Micromet & No & - \\
\hline 59 & 35 & 4 & No & Comedo & No & $\mathrm{BCS}$ & Macromet & Yes & $3 / 14$ \\
\hline
\end{tabular}


$B I-R A D S$ Breast Imaging Reporting and Data System, DCIS ductal carcinoma in situ, SLNB sentinel lymph node biopsy, $B C S$ breast-conserving surgery, $T M$ total mastectomy.

Table 3

Distribution of baseline features in patients with positive and negative SLNB

$\begin{array}{ccc}\text { Positive SLNB } & \text { Negative SLNB } & \text { Total } \\ (\mathrm{n}=13) & (\mathrm{n}=162) & (\mathrm{n}=175)\end{array} \quad p$ value

$\begin{array}{llll}\text { Age at diagnosis (years) } \quad 60.5( \pm 9,6) & 59.7( \pm 9.8) \quad 59.8( \pm 9.8) \quad 0.777\end{array}$

\section{BI-RADS}

5

$\begin{array}{cccc}0(0 \%) & 34(21 \%) & 34(19.4 \%) & 0.015 \\ 9(69.2 \%) & 114(70.4 \%) & 123(70.3 \%) & \\ 4(30.8 \%) & 14(8.6 \%) & 18(10.3 \%) & \end{array}$

\section{Mammographic}

\section{findings}

Microcalcifications

$\begin{array}{cccc}13(100 \%) & 156(96.3 \%) & 169(96.6 \%) & 0.779 \\ 0(0 \%) & 1(0.6 \%) & 1(0.6 \%) & \\ 0(0 \%) & 5(3.1 \%) & 5(2.8 \%) & \end{array}$

\section{Lesion extension}

Mean (mm)

$<20 \mathrm{~mm}$

$\geq 20 \mathrm{~mm}$

$\begin{array}{cccc}28.8( \pm 19.8) & 19.9( \pm 16.8) & 20.6( \pm 17.1) & 0.072 \\ 4(30.8 \%) & 106(65.4 \%) & 110(62.9 \%) & 0.017 \\ 9(69.2 \%) & 56(34.6 \%) & 65(37.1 \%) & \end{array}$

\section{Multifocal disease}

Yes

$\begin{array}{cccc}3(23.1 \%) & 11(6.8 \%) & 14(8 \%) & 0.037 \\ 10(76.9 \%) & 151(93.2 \%) & 161(92 \%) & \end{array}$

No

\section{Estrogen receptors}




$\begin{array}{lccc}\text { Positive } & 9(69.2 \%) & 132(81.5 \%) & 141(80.6 \%) \\ \text { Unknown } & 0(0 \%) & 3(1.8 \%) & 3(1.7 \%)\end{array}$

\section{Progesterone receptors}

Negative

Positive

Unknown

\section{Ki67 index}

$\leq 14 \%$

$>14 \%$

Unknown

\section{Grade on VABB}

DIN I

DIN II

DIN III

\section{Central necrosis}

Yes

No

\section{Type of DCIS}

Comedo

Cribriform

Papillary

Solid

Others/Mixed

Time between VABB and surgery (days)

\section{Upstaging to invasive carcinoma}

No

$10(76.9 \%)$

$152(93.8 \%)$

$162(92.6 \%) \quad 0.025$

Yes

$$
\begin{array}{ccc}
4(30.8 \%) & 46(28.4 \%) & 50(28.6 \%) \\
9(69.2 \%) & 112(69.1 \%) & 121(69.1 \%) \\
0(0 \%) & 4(2.5 \%) & 4(2.3 \%)
\end{array}
$$

$\begin{array}{cccc}4(30.8 \%) & 76(46.9 \%) & 80(45.7 \%) & 0.261 \\ 9(69.2 \%) & 82(50.6 \%) & 91(52 \%) & \\ 0(0 \%) & 4(2.5 \%) & 4(2.3 \%) & \end{array}$

$\begin{array}{cccc}1(7.7 \%) & 22(13.6 \%) & 23(13.1 \%) & 0.243 \\ 7(53.8 \%) & 109(67.3 \%) & 116(66.3 \%) & \\ 5(38.5 \%) & 31(19.1 \%) & 36(20.6 \%) & \end{array}$

$\begin{array}{llll}9(69.2 \%) & 74(45.7 \%) & 83(47.4 \%) & 0.102 \\ 4(30.8 \%) & 88(54.3 \%) & 92(52.6 \%) & \end{array}$

$46.1( \pm 20.5) \quad 45( \pm 21.5) \quad 45.1( \pm 21.4) \quad 0.86$

$$
3(23.1 \%) \quad 10(6.2 \%) \quad 13(7.4 \%)
$$

BI-RADS Breast Imaging Reporting and Data System, DCIS ductal carcinoma in situ, SLNB sentinel lymph node biopsy, $V A B B$ vacuum-assisted breast biopsy, $D I N$ ductal intraepithelial neoplasia.

*Comedo vs. other DCIS types 
Table 4

Univariate analysis for risk of positive SLNB

\begin{tabular}{|c|c|c|c|c|c|}
\hline & $\begin{array}{l}\text { Regression } \\
\text { coefficient }\end{array}$ & $\begin{array}{l}\text { Standard } \\
\text { Error }\end{array}$ & OR & $95 \% \mathrm{CI}$ & $p$ value \\
\hline BI-RADS 5 & 2.33 & 3.114 & 4.69 & $1.282-17.224$ & 0.02 \\
\hline Lesion size $\geq 20 \mathrm{~mm}$ & 2.33 & 2.654 & 4.25 & $1.255-14.447$ & 0.02 \\
\hline Multifocal disease & 1.94 & 3.001 & 4.12 & $0.987-17.174$ & 0.05 \\
\hline Comedo DCIS & 2.03 & 2.199 & 3.54 & $1.044-11.969$ & 0.04 \\
\hline $\begin{array}{l}\text { Upstaging to invasive } \\
\text { cancer }\end{array}$ & 2.06 & 3.351 & 4.56 & $1.080-19.249$ & 0.04 \\
\hline
\end{tabular}

BI-RADS Breast Imaging Reporting and Data System, OR odds ratio, CI confidence interval, DCIS ductal carcinoma in situ.

Table 5

Multivariate analysis for risk of positive SLNB

$\begin{array}{lccccc} & \begin{array}{c}\text { Regression } \\ \text { coefficient }\end{array} & \begin{array}{c}\text { Standard } \\ \text { Error }\end{array} & \text { OR } & 95 \% \text { CI } & p \text { value } \\ \text { BI-RADS 5 } & 1.36 & 2.067 & 2.76 & 0.637-11.977 & 0.17 \\ \text { Lesion size } \geq 20 ~ \mathbf{~ m m} & 1.12 & 1.605 & 2.23 & 0.546-9.136 & 0.26 \\ \text { Multifocal disease } & 1.98 & 4.259 & 5.14 & 1.015-26.066 & 0.048 \\ \text { Comedo DCIS } & 1.51 & 2.195 & 3.00 & 0.718-12.578 & 0.13\end{array}$


BI-RADS Breast Imaging Reporting and Data System, OR odds ratio, CI confidence interval, DCIS ductal carcinoma in situ. 